\title{
A Rare Case of Lipoleiomyoma of the Uterus
}

\author{
${ }^{1}$ Shuchi Lakhanpal, ${ }^{2}$ Manju Gupta, ${ }^{3}$ Anita Singla
}

\begin{abstract}
Lipoleiomyoma is an infrequent benign neoplasm of the uterus and is considered as a variant of uterine myomas. Their incidence varies from 0.03 to $0.2 \%$ in various reports. Lipoleiomyomas are made up of a varying proportion of mature adipocytes and smooth muscle cells. Lipoleiomyomas usually occur in asymptomatic perimenopausalor menopausal obese women. We, hereby, report a case of uterine lipoleiomyoma of the uterus.
\end{abstract}

Keywords: Benign tumor of uterus, Leiomyoma, Lipoleiomyoma.

How to cite this article: Lakhanpal S, Gupta M, Singla A. A Rare Case of Lipoleiomyoma of the Uterus, Journal of Postgraduate Medicine. Education and Research, Oct-Dec 2018;52(4):190-192.

\section{Source of support: Nil}

Conflict of interest: None

\section{INTRODUCTION}

Lipoleiomyomas or lipomatous tumors of the uterus are unusual benign neoplasms. ${ }^{1,2}$ This is a very rare lesion of the uterus occurring primarily in obese perimenopausal and postmenopausal women. The tumor consists of long intersecting bundles of smooth muscle cells amalgamated with foci of mature adipose cells and fibrous tissue. ${ }^{2-4}$ The aberrant presence of fatty tissue in the myometrium is termed as lipomatous degeneration of fibroid, smooth muscle metaplasia and also as lipoleiomyoma. It is usually a chance pathological finding in the postoperative specimen, and preoperative imaging can detect the location and lipomatous degeneration in fibroids. Lipoleiomyomas are benign tumors and do not increase the morbidity or mortality of the patient.

\section{CASE REPORT}

A 71-year-old lady presented to our outpatient department (OPD) with complaints of pain starting in the lower back and radiating to left leg for 3 months. She was a P2L2 postmenopausal lady with diabetes mellitus with hypertension and hypothyroid. Her sugar levels were controlled on drugs as was her hypertension. The patient

\footnotetext{
${ }^{1,2}$ Consultant, ${ }^{3}$ Associate Consultant

1-3Department of Obstetrics and Gynaecology, Jaypee Hospital, Noida, Uttar Pradesh, India
}

Corresponding Author: Shuchi Lakhanpal, Consultant, Department of Obstetrics and Gynaecology, Jaypee Hospital, Noida, Uttar Pradesh, India, e-mail: dr.shuchilkalia@gmail.com had a consultation with neurologist and neurosurgeon and received ilioinguinal and sacroiliac block, which relieved her pain. However, on USG whole abdomen, a leiomyoma like a mass of around $10 \times 6 \times 5 \mathrm{~cm}$ was identified, which was diagnosed as lipoleiomyoma of the uterus on computed tomography (CT) scan. It was considered that the pain could be because of compression of the ilioinguinal nerve by the enlarged lipoleiomyoma. Hence, the decision for hysterectomy was taken.

Gynecological examination (per vaginal) revealed an irregularly enlarged uterus corresponding to 12 weeks uterine size. Her fornices were free and non-tender.

All the standard blood and serological parameters were within normal range. The patient underwent total laparoscopic hysterectomy with bilateral salpingooophorectomy uneventfully. Postoperative period was also uneventful.

On gross examination of the specimen, the uterus measured $10 \times 7 \times 4 \mathrm{~cm}$ and had one intramural wellcircumscribed round mass, a large intramural fibroid measuring $7.5 \times 4.0 \times 5.0 \mathrm{~cm}$ (Figs 1 and 2). It was present on the lower uterine segment and looked typically like a uterine leiomyoma, but also had a pale yellow and a slightly softer consistency at its cut surface,with no gritty feel, unlike other fibroids. A polyp was identified obliterating endometrial cavity and measuring $2.5 \times 1.8 \times 1.5$ $\mathrm{cm}$. Another subserosal fibroid was identified measuring $3.7 \times 2.8 \times 2.5 \mathrm{~cm}$. The fallopian tubes appeared grossly normal. Bilateral ovaries were atrophic.

On histopathology, sections from intramural fibroid confirmed the presence of leiomyoma. A section from

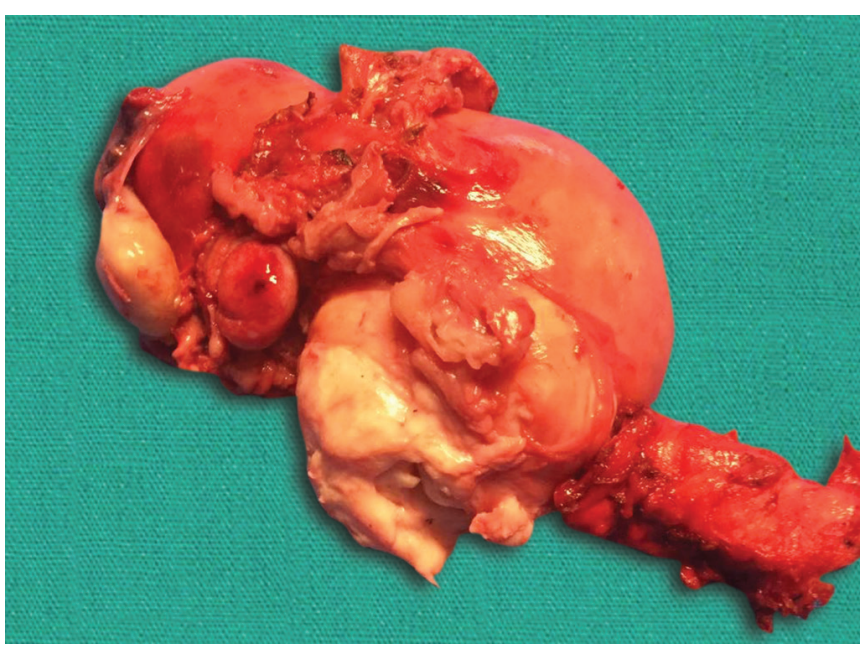

Fig. 1: Gross specimen showing uterus with cervix with large intramural fibroid measuring $7.5 \times 4.0 \times 5.0 \mathrm{~cm}$ 


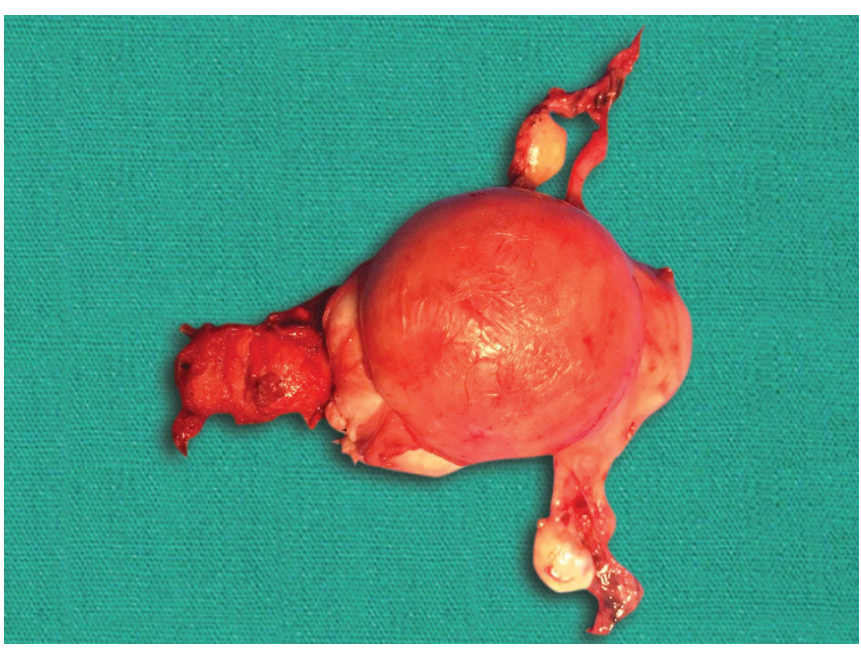

Fig. 2: Gross specimen showing lipoleiomyoma sitting on uterus

polyp protruding into the endometrial cavity showed features of the adenomyomatous polyp. Sections from subserosal fibroid showed features of lipoleiomyoma. There was no evidence of malignancy.

\section{DISCUSSION}

Lipoleiomyomais an unusual fatty tumor having predominant fatty components admixed with smooth muscles and fibrous tissues.

Myolipoma of soft tissue, as we know today, was first described in 1991 by Meis and Enzinger. The differential diagnosis of a lipomatous mass in the pelvic cavity could include benign cystic teratoma, malignant degeneration of a cystic teratoma, non-teratomatous lipomatous ovarian tumor, fibromyolipoma, benign pelvic lipoma, lipoblastic lymphadenopathy, and liposarcomas. Some association of lipomatous uterine tumors and endometrial carcinomas have been observed, and lipoleiomyo sarcoma that developed in uterine lipoleiomyomas has been expressed previously. ${ }^{5}$

Pathologically, the uterinelipomatoustumorscan be classified as follows:

1. Pure lipoma: These are constituted by mature fat cells. It is well encapsulated.

2. Lipoleiomyoma/fibromyolipoma/angiomyolipoma: It is made up of various mesodermal tissue components including adipose tissue, connective tissue, smooth muscles, and a fibrous component.

3. Liposarcoma: The rarest groupthat consists of sarcomatous change arising in less differentiated fat cells. ${ }^{6}$

Uterine lipoleiomyomais most commonly seen in postmenopausal women, at 50 to 70 years of age. It is a well-circumscribed tumor with a thin connective tissue capsule, located mostly in the posterior wall of the uterus.? Lipoleiomyomas may be single or multiple, ordinarily measuring around 5 to $10 \mathrm{~cm}$, extremes may go from few $\mathrm{mm}$ to up to $30 \mathrm{~cm}$. Most patients remain asymptomatic. Some may, however, experience symptoms similar to uterine myomas. This may include pelvic heaviness, pain, discomfort, pressure, abdominal lump and also vaginal bleeding. Rarely, they may present with postmenopausal bleeding. ${ }^{8}$ They may be seen in association with hyperestrogenic conditions like endometriosis, adenomyosis, endometrial hyperplasia, uterine polyps andcertain gynecological malignancies. ${ }^{9}$

Lipoleiomyomas can arise from any site in the uterus, in the cervix, the serosal layer or the broad ligament. Most commonly, it is located at uterine corpus, intramurally. As fatty tissue is not a part of the normal myometrium, the exact pathogenesis is obscure. Many theories such as fatty metamorphosis, lipomatous degeneration, lipomatous metaplasia in the smooth muscle cells, metaplasia in pericapillarypleuripotential mesenchymal cells, and perivascular extension of peritoneal or retroperitoneal fat along the blood vessels have been suggested. ${ }^{10,11}$

Precise identification of the uterine origin of the mass can be made by a transvaginal ultrasound. The lipomatous tumors show variable echogenicity on ultrasound. The typical appearance of uterine lipoleiomyoma is a well defined echogenic mass with a peripheral hypoechoic halo which represents compressed myometrium. The main ultrasound differential diagnosis of echogenic uterine mass is ovarian teratoma, especially when the ovary and the uterus are closely applied.

Imaging techniques including $\mathrm{CT} /$ magnetic resonance imaging (MRI) can help in determining the location and fatty nature of lipoleiomyomas, but mostly this is an incidental postoperative finding on histopathology. MRI with fat suppression sequence will delineate uterine lipoleiomyomas with high sensitivity and specificity to fat, and its multisectional ability will help recognize the location accurately. Predominant fat in a uterine mass on $\mathrm{CT}$ or MRI is considered diagnostic of lipoleiomyoma. ${ }^{12}$ Uterine lipoleiomyomas will routinely present as a welldemarcated hyperintense mass seen on $\mathrm{T} 1$ and $\mathrm{T} 2$ imaging, with hypointense amorphous bundles seen within.

Since lipoleiomyoma of the uterus is a rather rare tumor, a high degree of suspicion and knowledge of the imaging features of the same would help in making a correct diagnosis.

\section{CONCLUSION}

Lipoleiomyomas are rare benign uterine tumors with a similar clinical presentation as leiomyoma. Very rarely liposarcoma can arise from preexisting lipoleiomyoma. These tumors should be differentiated from fatty ovarian tumors, leiomyoma with degenerative changes, and uterine sarcomas. Though the management of this lesion is same as leiomyoma, the curiosity regarding its histogenesis 
continues. According to many studies, tumor metaplasia which is a well-recognized entity might explain the phenomena. To conclude, lipoleiomyoma of the uterus is a uniquely rare tumor with clinical presentation mimicking uterineleiomyomas, having innate radiological features, distinguishable histopathology, and an excellent prognosis.

\section{REFERENCES}

1. Lin KC, Sheu BC, Huang SC. Lipoleiomyoma of the uterus. Int J Gynaecol Obstet. 1999 Oct;67(1):47-49.

2. Rollasson TP, Wilkinson N. Non neoplastic conditions of myometrium and pure mesenchymal tumours of the uterus. In: Fox H, Wells M, editors. Haines and Taylor Obstetrical and gynaecological pathology. 5th ed. Edinburgh: Churchill Livingstone; 2003. p. 531.

3. Scurry JP, Carey MP, Targett CS, Dowling JP. Soft tissue lipoleiomyoma. Pathology. 1991 Oct;23(4):360-362.

4. Gentile R, Zarri M, De Lucchi F, Bellone F, Lapertosa G. Lipoleiomyoma of the uterus. Pathologica. 1996 Apr;88(2):132-134.
5. Lin M, Hanai J. Atypical lipoleiomyoma of the uterus. Acta Pathol Jpn. 1991 Feb;41(2):164-169.

6. Kitajima K, Kaji Y, Imanaka K, Sugihara R, Sugimura K. MRI findings of uterine lipoleiomyoma correlated with pathologic findings. AJR Am J Roentgenol. 2007 Aug;189(2):W100-104.

7. Deb S, Harith AK, Bhatnagar PK. Uterine Lipoma - A Rare Entity. Med J Armed Forces India. 2005 Oct;61(4):385-386.

8. Ghosh B, McKeown B, Gumma A. Lipoleiomyoma. BMJ Case Rep. 2011 Aug 31;2011.pii: bcr0820114577.

9. Akbulut M, Gündoğan M, Yörükoğlu A. Clinical and pathological features of lipoleiomyoma of the uterine corpus: a review of 76 cases. Balkan Med J. 2014 Sep;31(3):224-229.

10. Prieto A, Crespo C, Pardo A, Docal I, Calzada J, Alonso P. Uterine lipoleiomyomas: US and CT findings. Abdom Imaging. 2000 Nov-Dec;25(6):655-657.

11. Loffroy R, Nezzal N, Mejean N, Sagot P, Krausé D. Lipoleiomyoma of the uterus: imaging features. Gynecol Obstet Invest. 2008;66(2):73-75.

12. Tsushima $\mathrm{Y}, \mathrm{Kita} \mathrm{T}$, Yamamoto K. Uterine lipoleiomyoma: MRI, CT and ultrasonographic findings. Br J Radiol. 1997 Oct;70(838):1068-1070. 